\title{
Identification of the allosamidin-releasing factor in allosamidin-producing Streptomyces
}

\author{
Shigeo Suzuki, Hiromichi Nagasawa and Shohei Sakuda \\ The Journal of Antibiotics (2014) 67, 195-197; doi:10.1038/ja.2013.109; published online 30 October 2013
}

Keywords: Allosamidin; chitinase; Streptomyces

Allosamidin (Figure 1), a metabolite of Streptomyces, has a unique pseudotrisaccharide structure mimic to chitin ${ }^{1}$ and inhibits all 18 members of the chitinase family, ${ }^{2}$ which hydrolyze chitin and are widely present in nature. Allosamidin has been used to investigate the physiological role of chitinases involved in a variety of organisms including insects, ${ }^{3}$ yeasts ${ }^{4}$ and parasites. ${ }^{5}$ Recently, it was found that allosamidin and its derivative showed anti-asthmatic activity in a mouse model of asthma. ${ }^{6,7}$

In contrast, we have been seeking for a physiological role of allosamidin in its producer Streptomyces and found that allosamidin can markedly promote chitinase production and growth of its producer, Streptomyces sp. AJ9463, in a chitin medium. In the strain, the $46 \mathrm{kDa}$ chitinase, which was originated from chi65, was produced as the main chitinase in the culture filtrate. ${ }^{8}$ Production of the chitinase was strongly enhanced by a few hundred nano molar of allosamidin through a two-component regulatory system encoded by the two genes, chi65S and chi65R, present at the $5^{\prime}$-upstream region of the chitinase gene (Figure 1). ${ }^{9}$ It was shown that the promotion of the chitinase production by allosamidin was observed under the presence of $N, N^{\prime}$-diacetylchitobiose $\left[(\mathrm{GlcNAc})_{2}\right] .(\mathrm{GlcNAc})_{2}$ is the main product by the action of a chitinase on chitin and is also known as the general inducer for chitinase production in Streptomyces. ${ }^{10}$ $(\mathrm{GlcNAc})_{2}$ induced the production of the $46 \mathrm{kDa}$ chitinase originated from chi65 without using the two-component system of Chi65S and Chi65R in the strain AJ9463. ${ }^{9}$ Therefore, allosamidin may act on the two-component regulatory system after induction of the chitinase production by $(\mathrm{GlcNAc})_{2}$. Furthermore, we found a phenomenon that allosamidin was located in the mycelia of strain AJ9463 when cultured in a medium without chitin, but it was released into a culture filtrate from the mycelia when incubated in a chitin medium. ${ }^{8}$ These facts suggested that allosamidin responds to the chitin present in an environment such as soil and acts as a signal molecule for the promotion of chitinase production and growth in the environment in its producer Streptomyces.

The molecular mechanism how allosamidin is released from mycelia into outside of the cells in the presence of chitin is not clear, but it is speculated that chitin or its degradation product may act as a releasing factor in the process of the allosamidin release. Identification of the factor may afford an important clue to clarify the molecular mechanism for the allosamidin's action as a signal molecule. In this paper, we describe the identification of $(\mathrm{GlcNAc})_{2}$ as the releasing factor.

We previously showed that allosamidin adsorbed in the mycelia of strain AJ9463 was not released into a culture filtrate by incubating the mycelia in an inorganic salt medium but was released from the cells into the culture filtrate when the mycelia with allosamidin were incubated in the inorganic salt medium containing chitin. ${ }^{8}$ This inorganic salt medium system was used to assay the allosamidinreleasing activity in this study. Spores of the strain AJ9463 were inoculated into a Bennett medium consisting of $1 \%$ glucose, $0.2 \%$ peptone, $0.1 \%$ meat extract and $0.1 \%$ yeast extract, $\mathrm{pH} 7.2,(100 \mathrm{ml})$ in a $500-\mathrm{ml}$ Erlenmeyer flask, and the flask was incubated at $30^{\circ} \mathrm{C}$ and 150 r.p.m. on a rotary shaker for $72 \mathrm{~h}$. Mycelia were collected by centrifugation and washed with the inorganic salt medium consisting of $0.05 \% \mathrm{KCl}, 0.1 \% \mathrm{~K}_{2} \mathrm{HPO}_{4}, 0.05 \% \mathrm{MgSO}_{4}, 0.2 \%\left(\mathrm{NH}_{4}\right)_{2} \mathrm{SO}_{4}$ and $0.001 \% \mathrm{FeSO}_{4}, \mathrm{pH} 7.2$, three times. The washed mycelia containing allosamidin $(150 \mu \mathrm{g})$ were suspended in the inorganic salt medium $(50 \mathrm{ml})$. A volume of $1 \mathrm{ml}$ of the suspension was inoculated into the inorganic salt medium $(50 \mathrm{ml})$ containing each sugar sample and incubated at $30^{\circ} \mathrm{C}$ and 150 r.p.m. on a rotary shaker for $12 \mathrm{~h}$. The amounts of allosamidin in both the mycelia and supernatant were quantified by HPLC (column: Capcell-Pak $\mathrm{C}_{18}, 4.6 \times 250 \mathrm{~mm}$, Shiseido; mobile phase: gradient elution of $0-50 \% \mathrm{CH}_{3} \mathrm{CN}$ in $10 \mathrm{~mm} \mathrm{AcONH}_{4}-\mathrm{NH}_{4} \mathrm{OH}, \mathrm{pH}$ 8.9, in 30 min; flow rate: $1 \mathrm{ml} \mathrm{min}^{-1}$; detection: UV $220 \mathrm{~nm}$; retention time of allosamidin: $15.7 \mathrm{~min}$ ) according to the method previously described. ${ }^{11}$ The chitinase activity of the supernatant was measured using 4-methylumbelliferyl$N, N^{\prime}, N^{\prime \prime}$-triacetyl chitotrioside as a substrate. ${ }^{8}$

First, allosamidin-releasing activities of $\mathrm{N}$-acetylglucosamine and a mixture of chitooligosaccharides were measured in the inorganic salt medium system. Glucose and chitin were used as the negative and positive control, respectively. As a result, glucose and 


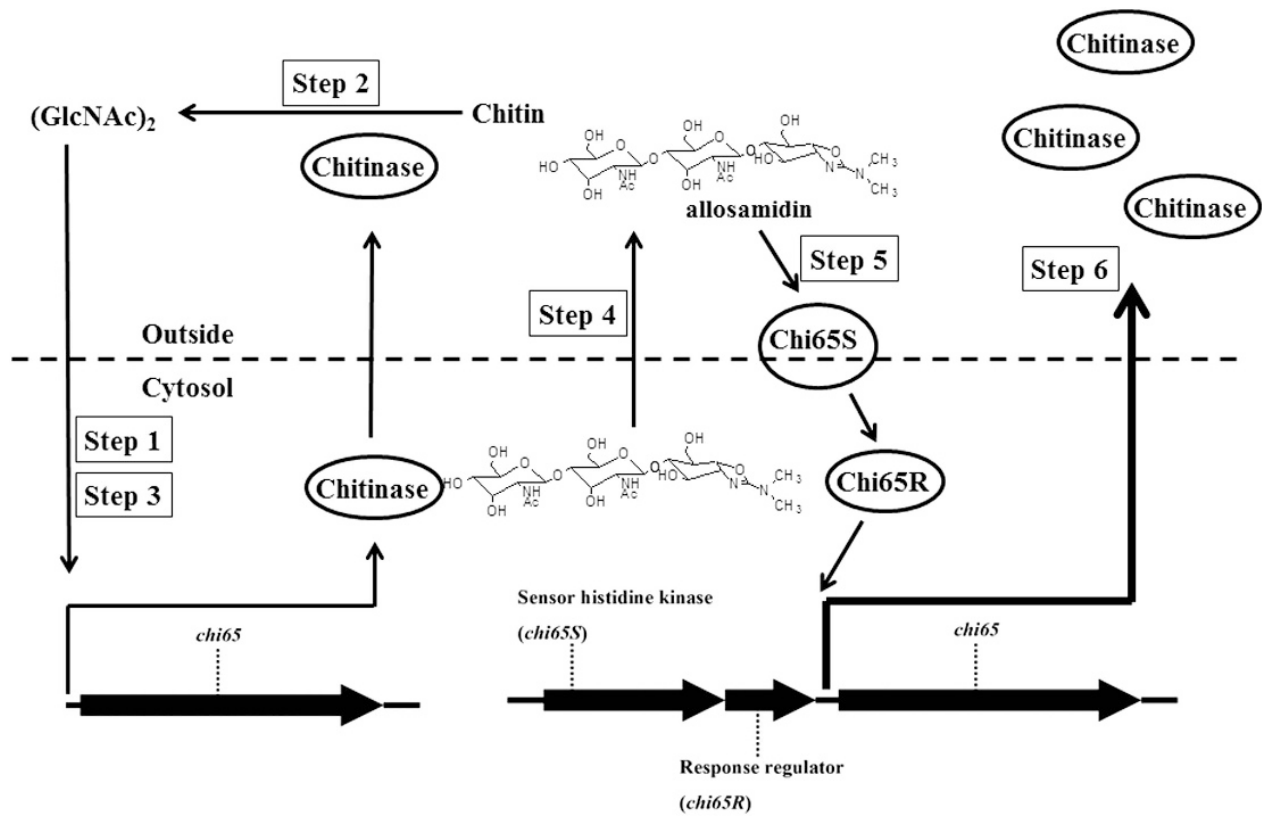

Figure 1 Allosamidin and chitinase production.
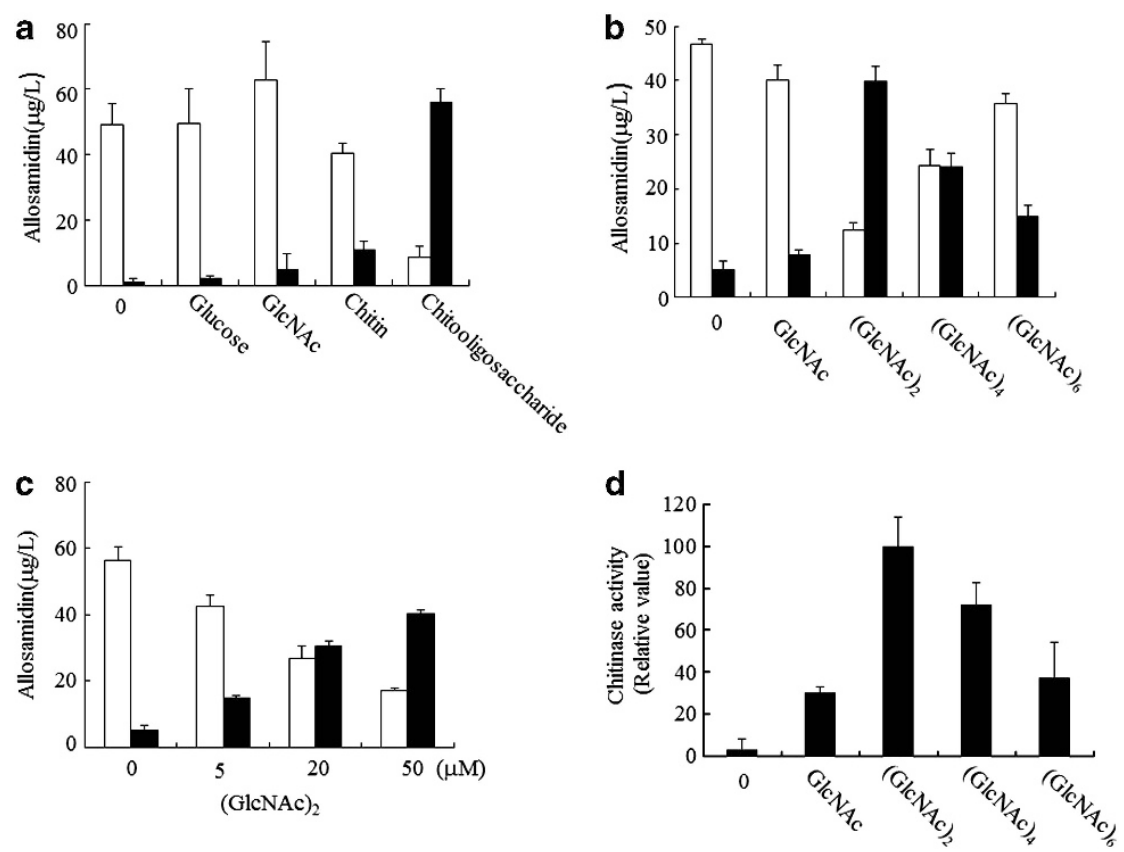

Figure 2 Effects of sugar samples on allosamidin release (a, b and $\mathbf{c}$ ) and chitinase production (d). Mycelia of the strain AJ9463 containing allosamidin were incubated in the inorganic salt medium containing $0.5 \%$ sugar sample (a and b) (12 mM of (GlcNAc) 2 in b) and $0,5,20$ or $50 \mu \mathrm{m}$ of $(\mathrm{GlcNAc})_{2}$ (c) for $12 \mathrm{~h}$. The amounts of allosamidin in the mycelia (white bars) and culture filtrate (black bars) were quantified by HPLC. The chitinase activity of the culture filtrate of the experiment in $\mathbf{b}$ was measured $(\mathbf{d})$. Means \pm s.d. were shown $(N=3)$.

$N$-acetylglucosamine did not show the releasing activity and a mixture of chitooligosaccharides showed much stronger releasing activity than that of chitin (Figure 2a). The dimer, tetramer and hexamer $\left((\mathrm{GlcNAc})_{2},(\mathrm{GlcNAc})_{4}\right.$ and $(\mathrm{GlcNAc})_{6}$, respectively) were next tested. Among them, $(\mathrm{GlcNAc})_{2}$ showed the strongest releasing activity (Figure $2 \mathrm{~b}$ ). It released allosamidin from the cells in a dosedepending manner (Figure 2c). Figure $2 \mathrm{~d}$ showed the chitinase activity of the culture filtrate obtained by the experiment in Figure $2 \mathrm{~b}$. The order of the activities of $(\mathrm{GlcNAc})_{2},(\mathrm{GlcNAc})_{4}$ and $(\mathrm{GlcNAc})_{6}$ for allosamidin release was parallel to that for chitinase production. These results indicated that (GlcNAc) $)_{2}$ acts as the allosamidin-releasing factor in the allosamidin-producing strain.

From the results obtained in this study, we can imagine the following story for allosamidin's action on chitinase production of the 
strain AJ9463. When the mycelia of the strain AJ9463 containing allosamidin are incubated in the chitin medium, a small amount of $(\mathrm{GlcNAc})_{2}$ may be produced by a weak chitinase activity involved in the inoculated mycelia, and it may induce the chi65 expression without using the two-component regulatory system (step 1 in Figure 1). The chitinase produced may be secreted from the cells and may degrade chitin to produce more amount of (GlcNAc) 2 (step 2 ), which further promotes chitinase production (step 3) and bacterial growth and induces allosamidin release (step 4). Allosamidin may be transferred to outside of the cells by attaching a chitinase or by an unknown mechanism induced by $(\mathrm{GlcNAc})_{2}$. Allosamidin or the allosamidin-chitinase complex may bind to the sensor moiety of Chi65S (step 5), leading to a marked enhancement of chi65 expression (step 6) and bacterial growth. It has been revealed that allosamidin can enhance chitinase production of not only allosamidin-producing Streptomyces but also allosamidin-non-producing Streptomyces. ${ }^{12}$ Therefore, allosamidin molecules released by $(\mathrm{GlcNAc})_{2}$ into an environment such as soil might affect the chitin metabolism and population of Streptomyces in the environment. Works to clarify mechanism for allosamidin release and to identify the ligand of the sensor as well as to investigate the role of allosamidin in nature are now in progress.

\section{ACKNOWLEDGEMENTS}

This work was supported by the Grant-in-Aid for the Japan Society for the Promotion of Sciences (20380066 and 23380062).
1 Sakuda, S., Isogai, A, Matsumoto, S., Suzuki, A. \& Koseki, K. The structure of allosamisin, a novel insect chitinase inhibitor, produced by Streptomyces sp. Tetrahedron Lett. 27, 2475-2478 (1986).

2 van Scheltinga, A. C. T. et al. Stereochemistry of chitin hydrolysis by a plant chitinase/ lysozyme and X-ray structure of a complex with allosamidin: evidence for substrate assisted catalysis. Biochemistry 34, 15619-15623 (1995).

3 Sakuda, S., Isogai, A., Matsumoto, S. \& Suzuki, A. Search for microbial insect growth regulators II. Allosamidin, a novel insect chitinase inhibitor. J. Antibiot. 40, 296-300 (1987).

4 Sakuda, S. et al. Effects of demethylallosamidin, a potent yeast chitinase inhibitor, on the cell division of yeast. Agric. Biol. Chem. 54, 1333-1335 (1990).

5 Shahabuddin, M., Toyoshima, T., Aikawa, M. \& Kaslow, D. C. Transmission-blocking activity of a chitinase inhibitor and activation of malarial parasite chitinase by mosquito protease. Proc. Natl Acad. Sci. USA 90, 4266-4270 (1993).

6 Zhu, Z. et al. Acidic mammalian chitinase in asthmatic Th2 inflammation and IL-13 pathway activation. Science 304, 1678-1682 (2004)

7 Matsumoto, T. et al. Demethylallosamidin, a chitinase inhibitor, suppresses airway inflammation and hyperresponsiveness. Biochem. Biophys. Res. Commun. 390, 103-108 (2009).

8 Suzuki, S. et al. Chitinase inhibitor allosamidin is a signal molecule for chitinase production in its producing Streptomyces. I. Analysis of the chitinase whose production is promoted by allosamidin and growth accelerating activity of allosamidin. J. Antibiot. 59, 402-409 (2006).

9 Suzuki, S. et al. Chitinase inhibitor allosamidin is a signal molecule for chitinase production in its producing Streptomyces. II. Mechanism for regulation of chitinase production by allosamidin through a two-component regulatory system. J. Antibiot. 59, 410-417 (2006).

10 Saito, A., Ishizaka, M., Francisco, P. B. Jr., Fujii, T. \& Miyashita, K. Transcriptional coregulation of five chitinase genes scattered on the Streptomyces coelicolor A3(2) chromosome. Microbiology 146, 2937-2946 (2000).

11 Zhou, Z. Y., Sakuda, S. \& Yamada, Y. Biosynthetic studies on the chitinase inhibitor, allosamidin. Origin of the carbon and nitrogen atoms. J. Chem. Soc. Perkin Trans. I 1992, 1649-1652 (1992).

12 Suzuki, S. et al. Chitinase inhibitor allosamidin promotes chitinase production of Streptomyces generally. Int. J. Biol. Macromol. 43, 13-19 (2008). 